\title{
CONTRACTIONS OF POISSON-LIE GROUPS, LIE BIALGEBRAS AND QUANTUM DEFORMATIONS
}

\author{
ANGEL BALLESTEROS \\ Departamento de Física, Universidad de Burgos \\ E-09003, Burgos, Spain \\ E-mail: angelb@trantor.cid.ubu.es \\ MARIANO A. DEL OLMO \\ Departamento de Física Teórica, Universidad de Valladolid \\ E-47011, Valladolid, Spain \\ E-mail: olmo@cpd.uva.es
}

\begin{abstract}
Contractions of Poisson-Lie groups are introduced by using Lie bialgebra contractions. As an application, contractions of $S L(2, R)$ Poisson-Lie groups leading to $(1+1)$ Poincaré and Heisenberg structures are analysed. It is shown how the method here introduced allows a systematic construction of the Poisson structures associated to non-coboundary Lie bialgebras. Finally, it is sketched how contractions are also implemented after quantization by using the Lie bialgebra approach.
\end{abstract}

1. Introduction. In a previous work [2], contractions of quantum algebras have been studied and classified by making use of the Lie bialgebra structure that underlies any quantum universal enveloping algebra. This paper completes those results by introducing, through the link between Poisson-Lie (PL) groups and Lie bialgebras stated in [5], a contraction method for PL groups.

Such a procedure turns out to be interesting from two different points of view: on one hand, as an efficient way to construct PL structures associated to non-coboundary Lie bialgebras, for which no Sklyanin bracket is available. On the other, as a first excursion in the field of contractions of general Poisson structures and their dynamical implications.

As a first step, we briefly review in Section 2 the basics of Lie algebra and Lie bialgebra contraction theories, with special emphasis in the ability of contractions to "generate

1991 Mathematics Subject Classification: 16W30, 17B37, 81R50.

This work has been partially supported by DGICYT (PB94-1115) and DGES (PB45-0719) projects from the Ministerio de Educación y Ciencia de España.

The paper is in final form and no version of it will be published elsewhere. 
cohomology". In Section 3 the PL contraction method is developed by making use of the results for Lie bialgebras given in the previous section and the duality between Lie group coordinates and Lie algebra generators. The resultant scheme is illustrated by means of two different PL $\operatorname{sl}(2, R)$ groups whose contractions lead to Heisenberg and Poincaré $(1+1)$ Poisson bivectors. Finally, the connection with the quantum algebra contraction procedure developed in [2] is outlined.

It is worth to be commented that, throghout the paper, Poisson bivectors are expressed in local coordinates, for which the contraction prescription is always well defined. The usual version of quadratic Sklyanin brackets can be recovered when the entries of a given matrix realization of the group are considered. We also emphasize the fact that, as far as contractions are concerned, first order terms in the deformation parameter seem to provide all the information needed to contract the whole quantum structure. It could be interesting to see whether this is or not a general property of quantum deformations and, among other open problems, how this approach can be applied to multiparametric PL groups and which are the physical implications of dynamical systems with PL symmetries.

2. Lie bialgebra contractions. After recalling the concept of Lie algebra contraction, firstly introduced in [6], the extension of the contraction procedure to Lie bialgebras is presented [2].

2.1. Lie algebra contractions. We shall present contractions by following the "universal" (representation independent) approach of [7].

Definition 1. Let $(A, m)$ and $\left(A^{\prime}, m^{\prime}\right)$ be two algebras with the same underlying vector space $V$ and products $m$ and $m^{\prime}$, i.e., $m: V \otimes V \rightarrow V$. Let us assume that there exists a continuous uniparametric family $\phi_{\varepsilon}$ of linear mappings

$$
\phi_{\varepsilon}: V \rightarrow V, \quad \varepsilon \in(0,1],\left.\quad \phi_{\varepsilon}\right|_{\varepsilon=1}=i d,
$$

such that $\phi_{\varepsilon}$ is invertible when $\varepsilon \neq 0$ and singular when $\varepsilon=0$. The algebra $\left(A^{\prime}, m^{\prime}\right)$ is said to be a contraction of $(A, m)$ if $m^{\prime}$ can be defined as

$$
m^{\prime}=\lim _{\varepsilon \rightarrow 0} m_{\varepsilon}=\lim _{\varepsilon \rightarrow 0} \phi_{\varepsilon}^{-1} \circ m \circ\left(\phi_{\varepsilon} \otimes \phi_{\varepsilon}\right) .
$$

Let us note that, when $A$ and $A^{\prime}$ are Lie algebras with Lie brackets $m$ and $m^{\prime},(2.2)$ can be written as

$$
[X, Y]^{\prime}:=\lim _{\varepsilon \rightarrow 0} \phi_{\varepsilon}^{-1}\left[\phi_{\varepsilon}(X), \phi_{\varepsilon}(Y)\right] .
$$

Definition 2. [8] Let $g$ be a Lie algebra whose associated vector space $V$ is written as a direct sum

$$
V=\bigoplus_{i} V_{i}, \quad i=0,1, \ldots, N \geq 1
$$

The mapping $\phi_{\varepsilon}$ will be called generalized Inönü-Wigner $(I W)$ contraction if

$$
\left.\phi_{\varepsilon}\right|_{V_{i}}=\left.\varepsilon^{n_{i}} I d\right|_{V_{i}}, \quad 0 \leq n_{0}<n_{1}<n_{2}<\ldots<n_{N}, \quad n_{i} \in R
$$

It can be shown that a given Lie algebra admits a generalized IW contraction if and only if

$$
\left[V_{i}, V_{j}\right] \subset \bigoplus_{k} V_{k}
$$


where by (2.6) we understand that a given subspace $V_{k}$ can give a contribution to the right hand side of the bracket if $n_{k} \leq n_{i}+n_{j}$.

EXAMPLE 3. Let us consider the three dimensional Lie algebra $s l(2, R)$, with commutation rules

$$
\left[J_{3}, J_{ \pm}\right]= \pm 2 J_{ \pm}, \quad\left[J_{+}, J_{-}\right]=J_{3} .
$$

The contraction mapping $\phi_{\varepsilon_{1}}$ defined by

$$
\phi_{\varepsilon_{1}}\left(J_{3}\right)=J_{3}, \quad \phi_{\varepsilon_{1}}\left(J_{+}\right)=\varepsilon_{1} J_{+}, \quad \phi_{\varepsilon_{1}}\left(J_{-}\right)=\varepsilon_{1} J_{-}
$$

gives rise, from $(2.3)$, to the brackets $\phi_{\varepsilon_{1}}^{-1} \circ m \circ\left(\phi_{\varepsilon_{1}} \otimes \phi_{\varepsilon_{1}}\right)$, i.e.,

$$
\left[J_{3}, J_{ \pm}\right]= \pm 2 J_{ \pm}, \quad\left[J_{+}, J_{-}\right]=\varepsilon_{1}^{2} J_{3},
$$

whose limit $\varepsilon_{1} \rightarrow 0$ leads to the defining relations of the $(1+1)$ Poincaré algebra.

In the same manner, let $\phi_{\varepsilon_{2}}$ be the contraction mapping

$$
\phi_{\varepsilon_{2}}\left(J_{3}\right)=\varepsilon_{2}^{2} J_{3}, \quad \phi_{\varepsilon_{2}}\left(J_{+}\right)=\varepsilon_{2} J_{+}, \quad \phi_{\varepsilon_{2}}\left(J_{-}\right)=\varepsilon_{2} J_{-} .
$$

The transformed brackets are now

$$
\left[J_{3}, J_{ \pm}\right]= \pm \varepsilon_{2}^{2} 2 J_{ \pm}, \quad\left[J_{+}, J_{-}\right]=J_{3},
$$

and the limit $\varepsilon_{2} \rightarrow 0$ gives the Heisenberg algebra as a contraction of $s l(2, R)$.

Contractions provide a very efficient way to obtain non-semisimple Lie algebras from semisimple ones. Among the two contractions introduced in the previous example, the former is called a simple IW contraction (there exist only the subspaces $V_{0}, V_{1}$ ), the latter is a generalized one.

2.2. Lie bialgebra contractions. We recall that a Lie bialgebra $(g, \eta)$ is a Lie algebra $g$ endowed with a cocommutator $\eta: g \rightarrow g \otimes g$ such that $\eta$ is a 1-cocycle and the dual map $\eta^{*}: g^{*} \otimes g^{*} \rightarrow g^{*}$ is a Lie bracket on $g^{*}$. A Lie bialgebra $(g, \eta)$ is called a coboundary bialgebra if there exists an element $\rho \in g \otimes g$ (the classical $r$-matrix), such that

$$
\eta(X)=[1 \otimes X+X \otimes 1, \rho], \quad \forall X \in g .
$$

It can be easily shown that the map (2.12), defined by means of an arbitrary $\rho$, is a Lie bialgebra if and only if the symmetric part of $\rho$ is invariant and the antisymmetric part of $\rho$ is a solution of the generalized Classical Yang-Baxter Equation [5].

The main statements relative to Lie bialgebra contractions are as follows.

TheOREM 4. [2] Let $(g, \eta)$ be a Lie bialgebra and let $g^{\prime}$ be a Lie algebra obtained by means of a (generalized) IW contraction $\phi_{\varepsilon}$ from $g$. If $n$ is a positive real number such that the limit

$$
\eta^{\prime}:=\lim _{\varepsilon \rightarrow 0} \varepsilon^{n}\left(\phi_{\varepsilon}^{-1} \otimes \phi_{\varepsilon}^{-1}\right) \circ \eta \circ \phi_{\varepsilon}
$$

exits, then $\left(g^{\prime}, \eta^{\prime}\right)$ is a Lie bialgebra. Moreover, there is a unique minimal value $f_{0}$ of $n$ such that, if $n \geq f_{0}$ the limit (2.13) exists, and if $n>f_{0}$ that limit is zero.

The crucial point in this result is that the number $f_{0}$ that ensures the existence of the limit (2.13) is uniquely defined. Moreover, $\eta^{\prime}$ turns out to be trivial when $n>f_{0}$. 
DEFINITION 5. If there exists a contraction $\phi_{\varepsilon}$ of $g$ to $g^{\prime}$ and a real number $n$ such that $\eta^{\prime}$ is given by $(2.13)$, the Lie bialgebra $\left(g^{\prime}, \eta^{\prime}\right)$ is called a contracted Lie bialgebra of $(g, \eta)$. The pair $\left(\phi_{\varepsilon}, n\right)$ will be called a Lie bialgebra contraction (or bicontraction). The minimal value $f_{0}$ that ensures the existence of $(2.13)$ will be called the fundamental contraction constant of $(g, \eta)$ associated to the contraction $\phi_{\varepsilon}$, and $\left(\phi_{\varepsilon}, f_{0}\right)$ will be a fundamental bicontraction.

A rather similar result can be obtained (in the case of coboundary Lie bialgebras) when the contraction of the classical $r$-matrix is considered:

THEOREM 6. [2] Let $(g, \eta(\rho))$ be a coboundary Lie bialgebra where $\rho$ is the classical $r$-matrix. Let $g^{\prime}$ be a Lie algebra obtained by means of a (generalized) IW contraction $\phi_{\varepsilon}$ from $g$. If $n$ is a positive real number such that the limit

$$
\rho^{\prime}:=\lim _{\varepsilon \rightarrow 0} \varepsilon^{n}\left(\phi_{\varepsilon}^{-1} \otimes \phi_{\varepsilon}^{-1}\right)(\rho)
$$

exists, then $\left(g^{\prime}, \eta^{\prime}\left(\rho^{\prime}\right)\right)$ is a coboundary Lie bialgebra. Moreover, there is a unique minimal value $c_{0}$ of $n$ such that, if $n \geq c_{0}$ (2.14) exists and, if $n>c_{0}$ such a limit is zero.

Complete proofs of theorems [4] and [6] can be found in [1]. In this last case we shall adopt the following terminology.

Definition 7. The minimal value $c_{0}$ that ensures the existence of the limit $(2.14)$ will be called coboundary contraction constant of the Lie bialgebra $(g, \eta(\rho))$ and relative to the contraction mapping $\phi_{\varepsilon}$. The pair $\left(\phi_{\varepsilon}, c_{0}\right)$ is called a coboundary bicontraction of $(g, \eta(\rho))$ linked to $\phi_{\varepsilon}$.

It is not difficult to prove that, for a given $(g, \eta(\rho))$ and $\phi_{\varepsilon}$, the relation $f_{0} \leq c_{0}$ is always fulfilled [1]. However, it must be emphasized that the expressions (2.13) and (2.14) are different and, in general, different results for the fundamental and coboundary contraction constants can be obtained. We shall take into account the following cases:

a) When $f_{0}=c_{0}$, the bicontraction $\left(\phi_{\varepsilon}, c_{0}=f_{0}\right)$ is coboundary and fundamental. Both the cocommutator $\eta^{\prime}$ and the contracted $r$-matrix $\rho$ are not trivial. As a consequence, $\left(g^{\prime}, \eta^{\prime}\left(\rho^{\prime}\right)\right)$ is a coboundary Lie bialgebra.

b) If $f_{0}<c_{0}$, the coboundary bicontraction $\left(\phi_{\varepsilon}, c_{0}=f_{0}\right)$ is not fundamental. Hence, the contracted $\rho^{\prime}$ will generate a trivial Lie bialgebra. On the contrary, the fundamental bicontraction $\left(\phi_{\varepsilon}, f_{0}\right)$ is not a coboundary one. Besides, as we shall see in the $\operatorname{sl}(2, R)$ case, the result of such a kind of contraction $\left(\phi_{\varepsilon}, f_{0}\right)$ will be a non-coboundary Lie bialgebra.

EXAMPLE 8. Let us consider the $s l(2, R)$ bialgebra $\left(s l_{2}, \eta(\rho)\right)$ given by the cocommutator

$$
\eta\left(J_{3}\right)=0, \quad \eta\left(J_{+}\right)=J_{+} \wedge J_{3}, \quad \eta\left(J_{-}\right)=J_{-} \wedge J_{3},
$$

which is generated by the classical $r$-matrix $\rho=J_{+} \wedge J_{-}$, that solves the modified CYBE. If we consider the contraction mapping $\phi_{\varepsilon_{1}}$ this coboundary bialgebra will be transformed by means of (2.13), and we shall easily find that the minimal $n$ that provides a well defined limit $\varepsilon_{1} \rightarrow 0$ (the fundamental contraction constant $f_{0}$ ) is 0 . So, in this case no "regularization" $\varepsilon_{1}^{f_{0}}$ is needed. Furthermore, it can be easily checked that the contracted 
cocommutator $\eta^{\prime}$ coincides with $\eta$, and defines a Lie bialgebra structure on the $(1+1)$ Poincaré algebra

$$
\left[J_{3}, J_{ \pm}\right]= \pm 2 J_{ \pm}, \quad\left[J_{+}, J_{-}\right]=0 .
$$

On the contrary, if we perform -by following (2.14)- the $\phi_{\varepsilon_{1}}$ contraction process on the classical $r$-matrix $\rho$ that generates this bialgebra, it turns out that the coboundary contraction constant $c_{0}$ is 2 : a factor $\varepsilon_{1}^{2}$ has to be included to avoid the divergency of (2.14) in the limit $\varepsilon_{1} \rightarrow 0$. This means that the fundamental bicontraction $\left(\phi_{\varepsilon_{1}}, n=f_{0}=0\right)$ is not a coboundary bicontraction, and the contracted bialgebra obtained from it is not expected to be a coboundary one. This is actually the case: there does not exist any element in $g^{\prime} \otimes g^{\prime}$ (with $g$ the algebra (2.16)) that originates $\eta^{\prime}$ in the form (2.12). On the other hand, if we consider the coboundary bicontraction $\left(\phi_{\varepsilon_{1}}, n=c_{0}=2\right)$, the contracted $r$-matrix $\rho^{\prime}$ exists, being again $\rho^{\prime}=J_{+} \wedge J_{-}$. However, the cocommutator that this element generates on the Poincaré algebra (2.16) is zero. This fact in accordance with Theorem 4 since $\left(\phi_{\varepsilon_{1}}, n=2\right)$ is not a fundamental bicontraction $\left(n>f_{0}\right)$.

EXAMPlE 9. The contraction mapping $\phi_{\varepsilon_{2}}$ (2.10) can be used to obtain, from the bialgebra $\left(s l_{2}, \eta(\rho)\right)$ and following the same steps as in the previous example, Heisenberg Lie bialgebra structures. The main point now is that computations lead to the same fundamental and coboundary contractions constants: $f_{0}=c_{0}=2$. Hence, the bicontraction $\left(\phi_{\varepsilon_{2}}, 2\right)$ originates a coboundary Heisenberg Lie bialgebra, where the contracted cocommutator $\eta^{\prime}$ coincides again with $\eta$ and the contracted $r$-matrix is $\rho^{\prime}=J_{+} \wedge J_{-}$.

EXAMPLE 10. Let us now consider a different $s l(2, R)$ (coboundary) bialgebra that we shall denote as $\left(s l_{2}, \eta_{+}\left(\rho_{+}\right)\right)$. It is usually called the non-standard one, defined by the cocommutator

$$
\eta_{+}\left(J_{+}\right)=0, \quad \eta_{+}\left(J_{3}\right)=J_{3} \wedge J_{+}, \quad \eta_{+}\left(J_{-}\right)=J_{-} \wedge J_{+} .
$$

The corresponding classical $r$-matrix is $\rho=J_{3} \wedge J_{+} / 2$, which is a solution of the CYBE. The analysis of the bicontractions induced by $\phi_{\varepsilon_{1}}$ and $\phi_{\varepsilon_{2}}$ can be summarized as follows:

$$
\phi_{\varepsilon_{1}}: f_{0}=1, \quad c_{0}=1 ; \quad \phi_{\varepsilon_{2}}: f_{0}=1, \quad c_{0}=3 .
$$

Note that the properties of these bicontractions are reversed when compared with the ones studied in Example 8: the $(1+1)$ non-standard Poincaré bialgebra is a coboundary one as a result of the $\left(\phi_{\varepsilon_{1}}, 1\right)$ contraction (moreover, $\rho^{\prime}$ coincides with $\rho$ ), and the fundamental bicontraction $\left(\phi_{\varepsilon_{2}}, 1\right)$ gives a non-coboundary Heisenberg structure. In both cases, the contracted cocommutator turns out to be (2.17) again.

3. Contractions of Poisson-Lie groups. There exists a one to one correspondence between PL structures on a (simply connected) Lie group $G$ and Lie bialgebras on $g=\operatorname{Lie}(G)[5]$. Given a PL group (i.e., a Poisson bracket $\Lambda$ on $C^{\infty}(G)$ for which the group multiplication is a Poisson map), let us write $\Lambda$ in local coordinates $\left(x^{1}, \ldots, x^{n}\right)$ as

$$
\Lambda=\pi^{i j}(x) X_{i}^{R} \wedge X_{j}^{R}, \quad(i<j) \quad i, j=1,2, \ldots, n,
$$

where $X_{i}^{R}$ denotes right invariant vector fields and $\pi^{i j}(x) \in C^{\infty}(G)$. Drinfel'd's assertion is that, if this Poisson bracket is compatible with the group multiplication and we identify 
the local coordinates with the generators of $g^{*}$, the bivector $\Lambda$ defines uniquely a Lie algebra $g^{*}$ with commutators $\left\{x^{i}, x^{j}\right\}=f_{k}^{i j} x^{k}$ where

$$
f_{k}^{i j}=\left.\frac{d}{d t}\left(\pi^{i j}\left(e^{t X_{k}}\right)\right)\right|_{t=0} .
$$

Furthermore, the Lie bracket given by the tensor $f$ is nothing but the dual $\eta^{*}$ of a cocommutator mapping that defines a Lie bialgebra $(g, \eta)$ that turns out to characterize the initial PL structure

$$
\eta\left(X_{n}\right)=f_{n}^{l m} X_{l} \otimes X_{m}
$$

Let $\phi_{\varepsilon}$ be a contraction mapping defined on the generators $X_{i}$ of a given Lie algebra $g$ and let $x^{j}$ be the dual (and local) coordinates of $G$ fulfilling

$$
\left\langle x^{i}, X_{j}\right\rangle=\delta_{j}^{i} \text {. }
$$

If $(\Phi(\varepsilon))_{i}^{j}$ is the matrix that realizes $\phi_{\varepsilon}$, on a given matrix realization of $g$ we shall have that the "contracted generators" will be

$$
\tilde{X}_{i}:=\phi_{\varepsilon}\left(X_{i}\right)=(\Phi(\varepsilon))_{i}^{j} X_{j} .
$$

Let us denote as $\psi_{\varepsilon}$ the contraction mapping that will act on the group coordinates in a form similar to $(3.5)$ :

$$
\tilde{x}^{i}:=\psi_{\varepsilon}\left(x^{i}\right)=(\Psi(\varepsilon))_{i}^{j} x^{j} .
$$

If we impose duality (3.4) to be preserved by the contraction process, i.e., that $\left\langle\tilde{x}^{i}, \tilde{X}_{j}\right\rangle=$ $\delta_{j}^{i}$, it is straightforward to check that the following relation must hold:

$$
\Psi(\varepsilon)=\left(\Phi(\varepsilon)^{-1}\right)^{T} .
$$

The contractions $\phi_{\varepsilon_{1}}$ and $\phi_{\varepsilon_{2}}$ are represented by diagonal $\Phi$ matrices and, in both cases, $\Psi(\varepsilon)=\left(\Phi(\varepsilon)^{-1}\right)$.

With all these prerrequisites, it is easy to translate the results on contractions of $\eta$ before obtained into the corresponding ones of the Lie bracket $\eta^{*}$. By dualizing (2.13) and substituting $\Phi(\varepsilon)$ we obtain (in the "diagonal" case) and for the fundamental bicontraction linked to a given $g$ and $\phi_{\varepsilon}$ :

$$
\eta^{* \prime}:=\lim _{\varepsilon \rightarrow 0} \varepsilon^{f_{0}} \Psi_{\varepsilon}^{-1} \circ \eta^{*} \circ\left(\Psi_{\varepsilon} \otimes \Psi_{\varepsilon}\right) .
$$

It is important to emphasize that, for a given PL group and a contraction mapping $\phi_{\varepsilon}$, all the elements in the expression (3.8) are uniquely defined as a consequence of Theorem 4: the constant $f_{0}$ is unique (in order to obtain a non trivial result) and the contraction $\Psi_{\varepsilon}$ of the coordinates is determined by $\phi_{\varepsilon}$. Therefore, the analysis of the linearized (3.2) PL bivector provided by the bicontraction suffices to obtain all the information relevant in order to contract the whole PL structure. This result can be stated as follows.

Proposition 11. Let $(G, \Lambda)$ be a PL group and let $\phi_{\varepsilon}$ be a contraction mapping defining a contraction from $g$ to $g^{\prime}$. If we assume that the contracted $P L$ group $\left(G^{\prime}, \Lambda^{\prime}\right)$ exists, the contracted $P L$ bracket is obtained, in local coordinates, through the formula

$$
\Lambda^{\prime}:=\lim _{\varepsilon \rightarrow 0} \varepsilon^{f_{0}} \psi_{\varepsilon}^{-1} \circ \Lambda \circ\left(\psi_{\varepsilon} \otimes \psi_{\varepsilon}\right),
$$

where $\psi_{\varepsilon}$ is given by (3.6) and $\left(\phi_{\varepsilon}, f_{0}\right)$ is the fundamental bicontraction of the Lie bialgebra $(g, \eta)$ associated to $(G, \Lambda)$. 
A relevant consequence of this Proposition arises when we consider, given a Lie bialgebra $(g, \eta)$, the problem of finding the PL bivector $\Lambda$ linked to it. There exists an answer to this question provided that $(g, \eta)$ is a coboundary bialgebra with classical $r$-matrix $\rho=\rho^{\alpha \beta} X_{\alpha} \otimes X_{\beta}$. In that case, $\Lambda$ is given by the so called Sklyanin bracket

$$
\Lambda=\rho^{\alpha \beta}\left(X_{\alpha}^{L} \otimes X_{\beta}^{L}-X_{\alpha}^{R} \otimes X_{\beta}^{R}\right),
$$

where $X_{i}^{L}\left(X_{i}^{R}\right)$ are left (right) invariant vector fields on $G$. The problem is that, if $(g, \eta)$ is a non-coboundary Lie bialgebra, no general expression playing the role of (3.10) has been found. However, from Proposition 11, we can give a contraction method to obtain non-coboundary bivectors by using the following corollary.

COROLlaRY 12. Let $\left(g^{\prime}, \eta^{\prime}\right)$ be a non-coboundary Lie bialgebra. If there exists another coboundary Lie bialgebra $(g, \eta(\rho))$ such that $\left(g^{\prime}, \eta^{\prime}\right)$ can be obtained as a (fundamental) bicontraction $\left(\phi_{\varepsilon}, f_{0}\right)$ of $(g, \eta)$, the PL group $\left(G^{\prime}, \Lambda^{\prime}\right)$ associated to $\left(g^{\prime}, \eta^{\prime}\right)$ can be obtained by applying $(3.9)$ to $(G, \Lambda)$.

Of course, $\Lambda$ can be always explicitly given by using (3.10). We recall that noncoboundary Lie bialgebras arise naturally within non-semisimple Lie algebras, like Poincaré, Galilei or Heisenberg ones [2]. We illustrate these results by using the Poisson counterparts of the Lie bialgebra contractions explained in previous examples.

EXAMPlE 13. To study this construction in the $s l(2, R)$ case, it is necessary to recall that the fundamental representation of its Lie algebra is given by

$$
D\left(J_{3}\right)=\left(\begin{array}{cc}
1 & 0 \\
0 & -1
\end{array}\right), \quad D\left(J_{+}\right)=\left(\begin{array}{ll}
0 & 1 \\
0 & 0
\end{array}\right), \quad D\left(J_{-}\right)=\left(\begin{array}{ll}
0 & 0 \\
1 & 0
\end{array}\right) .
$$

An arbitrary element of $S L(2, R)$ will be $g=e^{a_{-} J_{-}} e^{a_{+} J_{+}} e^{\chi J_{3}}$ where $\left(a_{+}, a_{-}, \chi\right)$ are local coordinates. In the representation (3.11) we shall have that $g$ will be written as

$$
D(g)=\left(\begin{array}{ll}
a & b \\
c & d
\end{array}\right)=\left(\begin{array}{cc}
e^{\chi} & a_{+} e^{-\chi} \\
a_{-} e^{\chi} & \left(1+a_{-} a_{+}\right) e^{-\chi}
\end{array}\right) .
$$

Now, left and right invariant vector fields can be obtained an read

$$
\begin{aligned}
X_{J_{+}}^{L} & =e^{2 \chi} \partial_{a_{+}}, \\
X_{J_{-}}^{L} & =a_{+}^{2} e^{-2 \chi} \partial_{a_{+}}+e^{-2 \chi} \partial_{a_{-}}+a_{+} e^{-2 \chi} \partial_{\chi}, \\
X_{J_{3}}^{L} & =\partial_{\chi} \\
X_{J_{+}}^{R} & =\left(1+2 a_{-} a_{+}\right) \partial_{a_{+}}-a_{-}^{2} \partial_{a_{-}}+a_{-} \partial_{\chi}, \\
X_{J_{-}}^{R} & =\partial_{a_{-}} \\
X_{J_{3}}^{R} & =-2 a_{-} \partial_{a_{-}}+2 a_{+} \partial_{a_{+}}+\partial_{\chi} .
\end{aligned}
$$

To perform contractions on the local coordinates, we have to define the $\psi_{\varepsilon}$ mappings. In particular, the one associated to $\phi_{\varepsilon_{1}}(2.8)$ is just its inverse, namely:

$$
\psi_{\varepsilon_{1}}\left(a_{+}\right)=\varepsilon_{1}^{-1} a_{+}, \quad \psi_{\varepsilon_{1}}\left(a_{-}\right)=\varepsilon_{1}^{-1} a_{-}, \quad \psi_{\varepsilon_{1}}(\chi)=\chi .
$$

Let us now recall the standard Lie bialgebra $(\operatorname{sl}(2, R), \eta(\rho))$ given by $(2.15)$. Since $\rho=$ $J_{+} \wedge J_{-}$and taking into account (3.13) and (3.14), the Sklyanin bracket (3.10) reads:

$$
\Lambda=-a_{+} \partial_{\chi} \wedge \partial_{a_{+}}-a_{-} \partial_{\chi} \wedge \partial_{a_{-}}-2 a_{-} a_{+} \partial_{a_{+}} \wedge \partial_{a_{-}} .
$$


In terms of the fundamental Poisson brackets (to which we shall refer from now on), we shall have

$$
\left\{\chi, a_{+}\right\}=-a_{+}, \quad\left\{\chi, a_{-}\right\}=-a_{-}, \quad\left\{a_{+}, a_{-}\right\}=-2 a_{-} a_{+} .
$$

As explained in Example 8., the bicontraction $\left(\phi_{\varepsilon_{1}}, f_{0}=0\right)$ was the fundamental one associated to the $s l(2, R) \rightarrow(1+1)$ Poincaré contraction. If we apply (3.9) to (3.17) and consider the mapping (3.15) we obtain that there is no $\varepsilon_{1}$ appearing in the transformed brackets $\varepsilon_{1}^{f_{0}} \psi_{\varepsilon_{1}}^{-1}\left(\left\{\psi_{\varepsilon_{1}}\left(x^{i}\right), \psi_{\varepsilon_{1}}\left(x^{j}\right)\right\}\right)$, and the limit $\varepsilon_{1} \rightarrow 0$ leads to the same fundamental Poisson brackets (3.17). Hence, when $\left(a_{+}, a_{-}, \chi\right)$ are considered as the light cone coordinates on the Poincaré group, (3.17) is the PL bracket linked to a non-coboundary bialgebra with cocommutator $\eta^{\prime}=\eta$ given by (2.15). Such fundamental brackets could not be obtained through (3.10), since for this bialgebra there exists no $r$-matrix $\rho$.

Example 14. Let us now consider the $s l(2, R) \rightarrow$ Heisenberg contraction defined by the mapping $\phi_{\varepsilon_{2}}$ (2.10) where the contracted coordinates will be given by the mapping

$$
\psi_{\varepsilon_{2}}\left(a_{+}\right)=\varepsilon_{2}^{-1} a_{+}, \quad \psi_{\varepsilon_{2}}\left(a_{-}\right)=\varepsilon_{2}^{-1} a_{-}, \quad \psi_{\varepsilon_{2}}(\chi)=\varepsilon_{2}{ }^{-2} \chi .
$$

The fundamental bicontraction was now $\left(\phi_{\varepsilon_{2}}, f_{0}=2\right)$. Therefore, we compute

$$
\begin{aligned}
\varepsilon_{2}{ }^{f_{0}} \psi_{\varepsilon_{2}}^{-1}\left(\left\{\psi_{\varepsilon}(\chi), \psi_{\varepsilon}\left(a_{ \pm}\right)\right\}\right) & =-a_{ \pm}, \\
\varepsilon_{2}{ }^{f_{0}} \psi_{\varepsilon_{2}}^{-1}\left(\left\{\psi_{\varepsilon}\left(a_{+}\right), \psi_{\varepsilon}\left(a_{-}\right)\right\}\right) & =-2 \varepsilon_{2}^{2} a_{-} a_{+} .
\end{aligned}
$$

In the limit $\varepsilon_{2} \rightarrow 0,(3.19)$ defines the bracket $\Lambda^{\prime}$; namely,

$$
\left\{\chi, a_{ \pm}\right\}^{\prime}=-a_{ \pm}, \quad\left\{a_{+}, a_{-}\right\}^{\prime}=0,
$$

that correspond to the coboundary Heisenberg bialgebra obtained in Example 9.

EXAMPLE 15. The same procedure can be straightforwardly applied to the nonstandard bialgebras $\left(g, \eta_{+}\right)$described in Example 10. The Sklyanin bracket on $S L(2, R)$ generated by the triangular $r$-matrix $\rho_{+}=J_{3} \wedge J_{+}$has the following brackets

$$
\left\{\chi, a_{+}\right\}=\left(e^{2 \chi}-1\right), \quad\left\{\chi, a_{-}\right\}=-a_{-}^{2}, \quad\left\{a_{+}, a_{-}\right\}=-2 a_{-}\left(1+a_{-} a_{+}\right) .
$$

The bicontraction $\left(\phi_{\varepsilon_{1}}, f_{0}=1\right)$ is the fundamental one and leads to the transformed brackets

$$
\begin{aligned}
\varepsilon_{1}{ }^{f_{0}} \psi_{\varepsilon_{1}}^{-1}\left(\left\{\psi_{\varepsilon_{1}}(\chi), \psi_{\varepsilon_{1}}\left(a_{+}\right)\right\}\right) & =\left(e^{2 \chi}-1\right), \\
\varepsilon_{1}{ }^{f_{0}} \psi_{\varepsilon_{1}}^{-1}\left(\left\{\psi_{\varepsilon_{1}}(\chi), \psi_{\varepsilon_{1}}\left(a_{-}\right)\right\}\right) & =-\varepsilon_{1}^{2} a_{-}^{2}, \\
\varepsilon_{1}{ }^{f_{0}} \psi_{\varepsilon_{1}}^{-1}\left(\left\{\psi_{\varepsilon_{1}}\left(a_{+}\right), \psi_{\varepsilon_{1}}\left(a_{-}\right)\right\}\right) & =-2 a_{-}\left(1+\varepsilon_{1}{ }^{2} a_{-} a_{+}\right),
\end{aligned}
$$

whose limit $\varepsilon_{1} \rightarrow 0$ is the (coboundary, since $\left.c_{0}=1\right)$ non-standard $(1+1)$ Poincaré PL structure [3] given by

$$
\left\{\chi, a_{+}\right\}=\left(e^{2 \chi}-1\right), \quad\left\{\chi, a_{-}\right\}=0, \quad\left\{a_{+}, a_{-}\right\}=-2 a_{-} .
$$

On the other hand, the contraction $\phi_{\varepsilon_{2}}$ can be performed on (3.21) by taking into account 
that $f_{0}=1$ and by using $\psi_{\varepsilon_{2}}(3.18)$. In this case we have that

$$
\begin{aligned}
\varepsilon_{2}{ }^{f_{0}} \psi_{\varepsilon_{2}}^{-1}\left(\left\{\psi_{\varepsilon_{2}}(\chi), \psi_{\varepsilon_{2}}\left(a_{+}\right)\right\}\right) & =\frac{\left(e^{2 \varepsilon_{2}{ }^{2} \chi}-1\right)}{\varepsilon_{2}{ }^{2}}, \\
\varepsilon_{2}{ }^{f_{0}} \psi_{\varepsilon_{2}}^{-1}\left(\left\{\psi_{\varepsilon_{2}}(\chi), \psi_{\varepsilon_{2}}\left(a_{-}\right)\right\}\right) & =-a_{-}^{2}, \\
\varepsilon_{2}{ }^{f_{0}} \psi_{\varepsilon_{2}}^{-1}\left(\left\{\psi_{\varepsilon_{2}}\left(a_{+}\right), \psi_{\varepsilon_{2}}\left(a_{-}\right)\right\}\right) & =-2 a_{-}\left(1+\varepsilon_{2}{ }^{2} a_{-} a_{+}\right) .
\end{aligned}
$$

And the limit $\varepsilon_{2} \rightarrow 0$ originates the Poisson brackets

$$
\left\{\chi, a_{+}\right\}=2 \chi, \quad\left\{\chi, a_{-}\right\}=-a_{-}^{2}, \quad\left\{a_{+}, a_{-}\right\}=-2 a_{-},
$$

that correspond to a non-coboundary (recall that $c_{0}=3$ ) Heisenberg Lie bialgebra already characterized in Example 10.

Two remarks can be made. Firstly, it is easy to check that the cocommutators of the Lie bialgebras involved here can be recovered from the obtained Poisson bivectors by applying (3.2). Secondly, note that, if we consider a PL group as a Poisson-Hopf algebra generated by the entries of a given matrix representation of $G$, the Sklyanin bracket defines a quadratic Poisson algebra. These new generators $(a, b, c, d$ in the case of $S L(2, R))$ are functions of the local coordinates used in our approach. In particular, if we consider the bivector (3.16) and the matrix representation (3.12), we obtain the following quadratic algebra

$$
\begin{array}{lll}
\{b, a\}=a b, & \{c, a\}=a c, \quad & \{c, b\}=0, \\
\{d, b\}=b d, & \{d, c\}=c d, \quad\{d, a\}=2 b c .
\end{array}
$$

In general, contractions cannot be implemented on such objects, since the mappings $\psi$ act "internally" on the entries $a, b, c, d$ through the local coordinates. This fact underlies the outstanding role that (before quantization and after it) the algebras (3.17) play. Actually, these algebras of fundamental Poisson brackets can be infinite dimensional (for instance, see $(3.21))$.

4. Quantization. If we consider an arbitrary quantum algebra $\left(U_{z}(g), \Delta_{z}\right)$, the first order term in $z$ of the deformed coproduct $\Delta_{z}$ always defines a Lie bialgebra structure on $g$, and the PL group associated to it is the classical counterpart of the quantum group dual to $\left(U_{z}(g), \Delta_{z}\right)$. It could be expected that the contraction scheme for Lie bialgebras and PL groups, here introduced, should be relevant to systematize "quantum contractions". In order to make explicit this connection, the role of the deformation parameter will be essential.

4.1. Contractions of uniparametric Lie bialgebras. Let us suppose that, given a determined (and perhaps coboundary) Lie bialgebra $(g, \eta(\rho))$, we introduce a uniparametric family of Lie bialgebras $(g, \delta(r))_{t}$ defined as

$$
\delta:=t \eta, \quad r:=t \rho .
$$

Two of these bialgebras will be said to be equivalent if there exists an automorphism of $g$ that transforms one into the other. In general, multiplication by $t$ can generate an infinite family of disequivalent structures. 
Let $\left(\phi_{\varepsilon}, f_{0}\right)$ be the fundamental bicontraction on $(g, \eta(\rho))$. Obviously, this bicontraction is also the fundamental one for the family $(g, \delta(r))_{t}$. However, if we consider the whole set of $t$-bialgebras, the definition of bicontraction can be rewritten by considering that the parameter $t$ is also transformed under the mapping $\phi_{\varepsilon}$ as

$$
\phi_{\varepsilon}(t):=\varepsilon^{-f_{0}} t \text {. }
$$

Under this assumption, it is easy to check that the contracted cocommutator $\delta^{\prime}$ can be defined by precluding explicitly the regularization factor $\varepsilon^{f_{0}}$ (which is now included in the transformation of $t$ ):

$$
\delta^{\prime}:=\lim _{\varepsilon \rightarrow 0}\left(\phi_{\varepsilon}^{-1} \otimes \phi_{\varepsilon}^{-1}\right) \circ \delta \circ \phi_{\varepsilon} .
$$

The same is true for the contracted $r$ matrix:

$$
r^{\prime}:=\lim _{\varepsilon \rightarrow 0}\left(\phi_{\varepsilon}^{-1} \otimes \phi_{\varepsilon}^{-1}\right)(r)
$$

Note that the inclusion of $t$ is also reflected at the PL group level, so, the new Poisson bracket is $\{,\}_{t}:=t\{$,$\} .$

4.2. Contractions of quantum algebras. In the frame of uniparametric quantum algebras, the parameter $t$ can be identified with the deformation parameter $z$, and the cocommutator $\delta$ is nothing but the (coantisymmetric) part of the first order $\Delta_{(1)}$ of the deformed coproduct $\Delta_{z}$. On the other hand, to contract a deformed Hopf algebra we shall have to define the transformation properties of the generators under $\phi_{\varepsilon}$ (the "classical" contraction) and, perhaps, some regularization that could depend of the order of a given term in the deformation parameter. In particular, this reasoning obviously implies that the contraction of its first order $\Delta_{(1)}$ has to be well defined.

This (necessary) condition is equivalent (in the case of coantisymmetric $\Delta_{(1)}$ ) to impose a non-trivial (fundamental) bicontraction on the associated family of Lie bialgebras $(g, \delta)$. But, as we have already demostrated, a fundamental bicontraction of any Lie bialgebra defines uniquely the $f_{0}$ constant which, in turn, induces a unique possible transformation (4.2) of the $t \equiv z$ parameter. This is the reason why the transformation of the deformation parameter under contraction (firstly introduced in [4]) provides a systematic and well-defined procedure to contract any quantum algebra. Thus,

DeFinition 16. Let $\left(U_{z}(g), \Delta_{z}, m_{z}\right)$ be a quantum algebra and let $(g, \delta)$ be its associated Lie bialgebra. We shall say that $\left(U_{z}\left(g^{\prime}\right), \Delta_{z}^{\prime}, m_{z}^{\prime}\right)$, with bialgebra $\left(g^{\prime}, \delta^{\prime}\right)$, is a quantum contraction of $\left(U_{z}(g), \Delta_{z}, m_{z}\right)$ if there exists a fundamental bicontraction $\left(\phi_{\varepsilon}, f_{0}\right)$ of $(g, \delta)$ such that

$$
\begin{aligned}
\Delta_{z}^{\prime} & :=\lim _{\varepsilon \rightarrow 0}\left(\phi_{\varepsilon}^{-1} \otimes \phi_{\varepsilon}^{-1}\right) \circ \Delta_{z} \circ \phi_{\varepsilon}, \\
m_{z}^{\prime} & :=\lim _{\varepsilon \rightarrow 0} \phi_{\varepsilon}^{-1} \circ m_{z} \circ\left(\phi_{\varepsilon} \otimes \phi_{\varepsilon}\right),
\end{aligned}
$$

where $\phi_{\varepsilon}(z)=\varepsilon^{-f_{0}} z$.

The same reasoning applies to quantum groups, since their first order commutation rules (in both the generators and $z$ ) will be given by the dual Lie bialgebras $\delta^{*}$, whose non trivial contraction is guaranteed by the fundamental constant $f_{0}$ and, therefore, by the transformed $z$. This method has been systematically used in [2] to contract quantum 
orthogonal algebras, and can be straightforwardly applied to the quantum deformations linked with the $\operatorname{sl}(2, R)$ bialgebras here studied.

\section{References}

[1] A. Ballesteros, Contractions of Lie bialgebras and quantum deformations of kinematical symmetries, Ph. D. Thesis (in Spanish), Universidad de Valladolid (1995).

[2] A. Ballesteros, N.A. Gromov, F.J. Herranz, M.A. del Olmo and M. Santander, Lie bialgebra contractions and quantum deformations of quasi-orthogonal algebras, J. Math. Phys. 36 (1995), 5916.

[3] A. Ballesteros, F.J. Herranz, C.M. Pereña, M.A. del Olmo and M. Santander, Non standard quantum $(1+1)$ Poincaré group: a T matrix approach, J. Phys. A: Math. Gen. 28 (1995), 7113.

[4] E. Celeghini, R. Giachetti, E. Sorace and M. Tarlini, Contractions of quantum groups, Lecture Notes in Mathematics n. 1510. Springer-Verlag, Berlín (1992) 221.

[5] V.G. Drinfel'd, Hamiltonian structures on Lie groups, Lie bialgebras and the geometric meaning of the classical Yang-Baxter equations, Sov. Math. Dokl. 27 (1983), 68.

[6] E. Inönü and E.P. Wigner, Contractions of groups and representations, Proc. Natl. Acad. Sci. U. S. 39 (1953), 510.

[7] E.J. Saletan, Contractions of Lie groups, J. Math. Phys 2 (1961), 1.

[8] E. Weimar-Woods, The three-dimensional real Lie algebras and their contractions, J. Math. Phys 32 (1991), 2028. 